\title{
Petri Net Based Modeling and GA Based Scheduling for a Flexible Manufacturing System
}

\author{
Yung-Yi Chung, Li-Chen Fu, and Ming-Wei Lin \\ Department of Computer Science and Information Engineering \\ National Taiwan University, Taipei, Taiwan, R.O.C.
}

\begin{abstract}
In this paper, a genetic algorithm (GA) embedded adaptive scheduling over a timed place Petri net (TPPN) model provides a new method for a flexible manufacturing system (FMS). The chromosome representation of the search nodes is constructed directly from the TPPN model of an FMS. A TPPN based schedule builder receives a chromosome and an initial marking as input, and then produces a near-optimal schedule.
\end{abstract}

\section{Introduction}

In this paper, we develop a TPPN modeling approach for FMSs, and implement it as an automatic Petri net generator (APNG) $[3,2,4]$. The APNG developed here has a graphic user interface that facilitates users to input the specification of an FMS, based on which the operator can create a TPPN model of the FMS. In addition, a GA search based adaptive scheduling method is also proposed to find an effective schedule $[5,2,4]$.

\section{TPPN Model Generator}

The hierarchical view of the entire system model contains two major sub-models [4]. One is called Transportation Model and the other is called Process-Flow Model. The objective of the Transportation Model is to model the behavior of the material handling devices traveling from the current stop to its destination stop, and that of the Process-Flow Model is to describe the behavior of parts routing and resources assignment. Each sub-model interacts with the other by using some common places. For example, when Job $n$ needs to call AGV $a$ to move to a specified stop $i$, the Process-Flow Model of Job $n$ will add a token in the place $m v \_i_{-} a$, which is also a place in the Movement Control Module of AGV $a$ of the Transportation Model as a command. When AGV $a$ arrives at the specified stop $i$, the Movement Control Module of AGV $a$ will place a token in the place at $i_{-} a$, which is also a place in the Process-Flow Model of Job $n$ to acknowledge that AGV $a$ just arrives at the specified stop.

\section{Genetic Algorithm Scheduler}

\section{Genetic Algorithm Scheduling}

\section{Schedule Builder}

A schedule builder is dedicated to transforming a chromosome to a feasible schedule wherely, we can evaluate the aforementioned indirect chromosome representation. Based on a TPPN model of the system, the evolution of the system can be described by the change of marking in the net. So, given a TPPN model and a chromosome, the schedule builder can generate a feasible schedule in terms of the firing sequence of transitions in the timed place Petri net model according to the chromosome. The firing sequence of transitions provides the order of the initiation of operations.

\section{Fitness Function}

The fitness value of chromosome can be determined after the schedule builder generates the schedule.

In the current implementation, we use the makespan, due date, and machine utilization as the performance measure, which are represented by $f_{1}$, $f_{2}$ and $f_{3}$, respectively. Then, we use linear scaling method to scale the value between 100 and 0 and get the scaled value $f_{1}^{\prime}$ to $f_{3}^{\prime}$.

Finally, we define the fitness values for each chromosome as follow:

$$
f_{i}=w_{1} \cdot f_{1}^{\prime}+w_{2} \cdot f_{2}^{\prime}+w_{3} \cdot f_{3}^{\prime}
$$

where $w_{1}$ to $w_{3}$ is predefine weighted number.

\section{Genetic Operations}

Reproduction In the implementation, we use the scheme where the probabilities of selection are calculated in the form:

$$
\text { pselect }_{i}=\frac{f_{i}}{\Sigma f_{i}}
$$




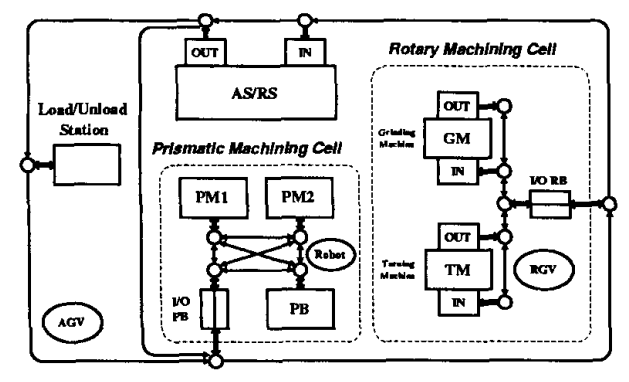

Figure 1: The System Layout.

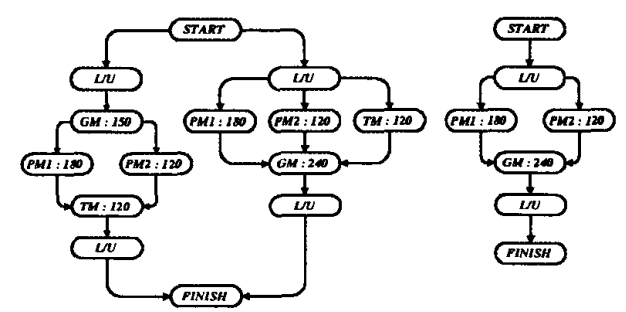

Figure 2: The Process Flow Graph of Job1 \& Job2.

- Then, the actrual number of chromosome $c_{i}$ is calculated by spinning the weighted roulette wheel.

Crossover The chromosome representation described above contains information about plan selections, resource assignments of every operation, and resource competition resolutions. We can create new chromosomes by exchanging portions of two old chromosomes simply.

\section{Adaptive Scheduling}

We use the implementation of the proposed adaptive scheduler [1]. We define WIP and the condition for the schedule generator, which generates the partial schedule in the current segmented GA search for every job. Although, the scheduling strategy may not provide an optimal schedule, when the number of parts to be processed is very high, it can generate an effective schedule in a shorter time interval.

\section{Experiment Results}

\section{System Layout and Process Flow}

This example illustrates a prototype FMS. The layout of this target system is shown in Figure 1. And Figure 2 shows the process flows of the two jobs.

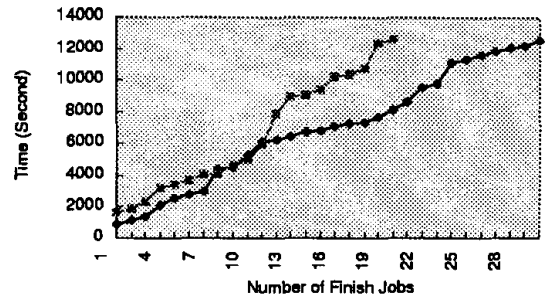

Figure 3: History of Job1 \& Job2.

\section{Implementation Results}

In Figure 3, the relation between the number of completed parts and the completion time is shown. In average, GA embedded search takes about 9 minutes for one generation on our PC.

\section{Conclusion}

In this paper, we consider the FMS scheduling problem. We first propose a systematic Petri net modeling for an FMS. The entire TPPN model is composed of two sub-models, one is Transportation Model and the other is Process Flow Model. Then, we propose an adaptive scheduling method incorporating the GA search. It not only generates an efficient schedule but also allows one to set the different priorities among the jobs.

\section{References}

[1] Carlos F. G. Bispo, João J. S. Sentieiro, and Roger D. Hibberd. Adaptive scheduling for highvolume shops. IEEE Trans. on Robotics and Automation, 8(6):696-706, December 1992.

[2] Doo Yong Lee and Frank DiCesare. Integrated models for scheduling flexible manufacturing systems. In Proc. IEEE Int. Conf. on Robotics and Automation, pages 827-832, 1993.

[3] James Lyle Peterson. Petri Net Theory and the modeling of Systems. Prentice-Hall, Englewood Cliffs, N.J., 1981.

[4] Tien-Hsiang Sun, Chao-Weng Cheng, and Li-Chen Fu. A Petri net based approach to modeling and scheduling for an FMS and a case study. IEEE Trans. on Industrial Electronics, 41(6):593-600, December 1994.

[5] Takeshi Yamada and Ryohei Nakano. A Genetic Algorithm applicable to large-scale job shop problems. In Parallel Problem Solving from Nature, volume 2, pages 281-290, 1992. 